\title{
Comparison of SOGI-FLL with SOGI-PLL for Single-Phase Grid- Connected Inverters
}

\author{
Royan $^{1 *}$, Trias Andromeda ${ }^{2}$, Mochammad Facta ${ }^{2}$, Hermawan $^{2}$, and Iwan Setiawan ${ }^{2}$ \\ ${ }^{1}$ Magister Program of Electrical Engineering, Diponegoro University, Semarang - Indonesia \\ ${ }^{2}$ Department of Electrical Engineering, Diponegoro University, Semarang - Indonesia
}

\begin{abstract}
Phase locked loop (PLL) is a feedback system that has a vital role in the grid-connected inverter systems. The primary function of PLL is to obtain various grid information, such as magnitude, frequency, and phase. That used as the basis for the synchronization process. The primary purpose of this paper is to model and compare the performance SOGI-FLL (Second Order Generalized Integrator Frequency Locked Loop) With SOGI-PLL, which is a relatively standard PLL. Besides that, this paper also aims to investigate the effect of FLL feedback gain on synchronization performance. Based on the simulation results, the performance of SOGI-FLL in its steady state is superior to that of SOGI-PLL especially for the condition of a single phase power systems that change in frequency.
\end{abstract}

Keywords: Phase locked loop; Second Order Generalized Integrator; SOGI-PLL; SOGI-FLL.

\section{Introduction}

Grid Inverters are one of the essential components in renewable power generation that connected to the grid. In recent years, grid inverters have been widely applied in single phase systems, such as on-grid PV systems [13].

Grid connection requirements, the inverter must continue to operate and be connected to the grid even though the grid is experiencing interference, such as damage to grid devices, connection, and interconnection of electrical loads, and voltage inter-phase imbalances [4].

Grid inverter system must have the ability to detect interference quickly, so that information on grid parameters such as the phase and magnitude needed by the on grid control inverter system are still available accurately.

An essential component in a grid inverter system that is responsible for providing information on magnitude, phase and frequency is PLL.

The PLL method has advantages in terms of simplicity, and the resulting output is relatively useful for detecting voltage signal parameters, so it is widely applied in inverter components. In addition to inverters, PLL is also widely applied in the electrical field, such as telecommunications systems, electric power systems, motor control systems, and so on.

Based on its structure, there are various PLL singlephase such as inverse park transform [5, 6], SOGI [7-9], Hilbert Transform [10-12], T/4 delay PLL, and Enhanced PLL [13]. In general, the main difference between PLL topology is how orthogonal signals generated by the orthogonal signal generator (OSG).

The main task of the PLL structure is to provide grid parameter references and monitor it, to ensure that the performance of the grid-connected system meets the standard requirements, if the instantaneous voltage on the grid rises or falls, the inverter output voltage must also follow. Likewise, if the frequency on the grid rises or falls, then the inverter output frequency must also follow [14].

This paper model and compares the performance of SOGI-FLL with SOGI-PLL, and investigates the effect of FLL feedback amplifiers on synchronization performance. The software used in this study is Matlab / Simulink

\section{SOGI-PLL Model}

One of the standard PLL types commonly used to detect phase angles, the magnitude of the voltage and frequency is SOGI-PLL as shown in Fig. 1.

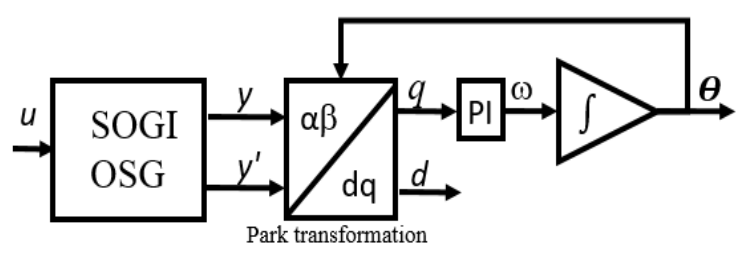

Fig. 1. SOGI-OSG based PLL

The input signal is $u$ wave and two sine output signals ( $y$ and $\left.y^{\prime}\right), y$ and $y^{\prime}$ ideally different phases of $90^{\circ}$

\footnotetext{
* Corresponding author: royan.belix@gmail.com
} 
so easily transformed in a park transformation[15]. The SOGI-OSG structure in continuous time-domain models presented in Fig. 2, and was defined in Eq. (1) to Eq.(3) [16].

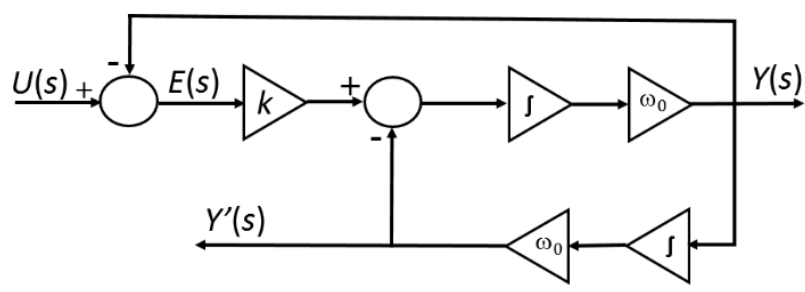

Fig. 2. SOGI-OSG in continuous time-domain

$$
\begin{aligned}
& \frac{Y(s)}{U(s)}=\frac{k \omega_{0} s}{s^{2}+k \omega_{0} s+\omega_{0}^{2}}, \\
& \frac{Y^{\prime}(g)}{U(s)}=\frac{k \omega_{0}{ }^{2}}{s^{2}+k \omega_{0} s+\omega_{0}^{2}}, \\
& \frac{E(s)}{U(s)}=\frac{s^{2}+\omega_{0}^{2}}{s^{2}+k \omega_{0} s+\omega_{0}^{2}} .
\end{aligned}
$$

Where $U(s)$ is an input signal while $Y(s)$ and $Y^{\prime}(s)$ are output signals, Signal $E(s)$ is the difference between input $U(s)$ and output $Y(s)$. The parameter $k$ amplifying error signal $E(s)$ affects the transient response. The SOGI-OSG structure presented in Fig. 2 has a wave-like output presented in Fig. 3.

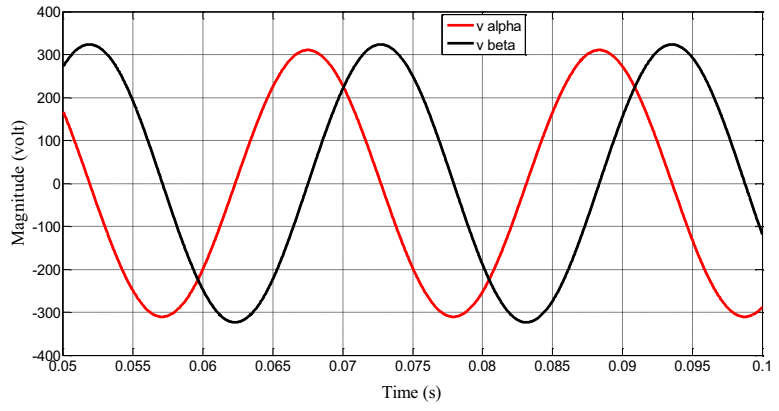

Fig. 3. Orthogonal voltage generator system $y[n]$ dan $y^{\prime}[n]$

$v_{a}$ (alpha) represents $Y(s)$ and $v_{\mathrm{B}}$ (beta) represents $Y^{\prime}(n)$ in the SOGI-OSG output. Then the park transformation represents the fixed frame of reference $(\alpha \beta)$ produced by the SOGI-OSG system into a moving frame of reference $d q . v_{d}$ represents the voltage magnitude, and $v_{q}$ denotes a vector of that magnitude, the value $v_{q}$ should be close to zero. Transformation will give the value $v_{d}$ and $v_{q}$ was defined in Eq. (4) to Eq.(5).

$$
\begin{aligned}
& {\left[\begin{array}{l}
v_{q} \\
v_{d}
\end{array}\right]=\left[\begin{array}{cc}
\cos \theta & -\sin \theta \\
\sin \theta & \cos \theta
\end{array}\right]\left[\begin{array}{l}
v_{a} \\
v_{\mathrm{d}}
\end{array}\right]} \\
& {\left[\begin{array}{l}
v_{a} \\
v_{\mathrm{B}}
\end{array}\right]=\left[\begin{array}{cc}
\cos \theta & \sin \theta \\
-\sin \theta & \cos \theta
\end{array}\right]\left[\begin{array}{l}
v_{q} \\
v_{d}
\end{array}\right]}
\end{aligned}
$$

Then the output of the park transformation will be given a PI control to get the frequency and phase values of the actual grid.

\section{SOGI-FLL Model}

SOGI-FLL is a type of PLL which is the result of the development of SOGI-PLL. The SOGI-PLL described in the previous section has one major disadvantage, when the input signal frequency is outside the $50 \mathrm{~Hz}$ power grid operational standard, SOGI output loses tracking accuracy. Frequency feedback will provide an essential role for SOGI control, so grid parameter estimates remain accurate. FLL gain $(\gamma)$ affects transient waves. The SOGI-FLL structure in continuous time-domain models presented in Fig. 4 [16].

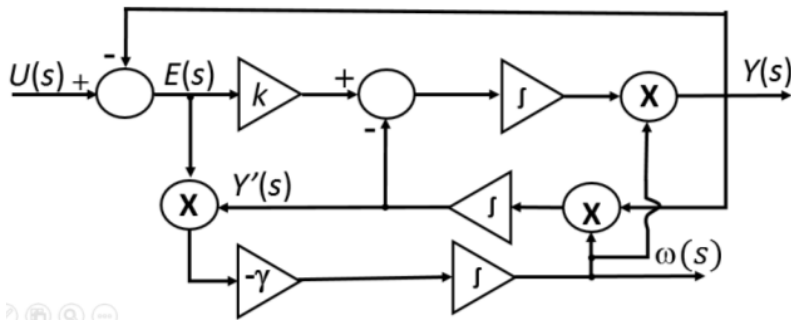

Fig. 4. SOGI-FLL in continuous time-domain

\section{Simulation Results and Discussion}

In this paper, the investigation of PLL performance is carried out by simulation studies. The software used in this study is Matlab/Simulink. Performance investigations include PLL responses to changes in frequency and magnitude of single-phase power input and investigating the effect of FLL feedback amplifiers on synchronization performance. The PLL structure presented has several parameters, as shown in Table 1.

Table 1. PLL Parameters

\begin{tabular}{|c|c|c|}
\hline Parameters & SOGI-PLL & SOGI-FLL \\
\hline$K$ & 1,2 & 1,2 \\
\hline$K p$ & 1 & 1 \\
\hline$K i$ & 1 & 1 \\
\hline$w_{0}$ & $2 \pi(50)$ & $2 \pi(50)$ \\
\hline$\gamma$ & - & -1 \\
\hline
\end{tabular}

\subsection{The performance of PLL against grid magnitude changes}

Fig. 5 shows a single phase grid magnitude profile with a frequency of $50 \mathrm{~Hz}$ which will be detected by SOGIPLL and SOGI-FLL. Based on the figure, that from 0 to 0.16 seconds the magnitude of the grid is worth 311 volts $(220 \sqrt{2})$, while from seconds to 0.16 to 0.24 seconds the magnitude decreases to 150 volts, while from seconds to 0.25 , the magnitude returns to normal. The estimation of the voltage, frequency and phase magnitudes by these PLLs is shown in Fig. 6 to Fig. 8 . 


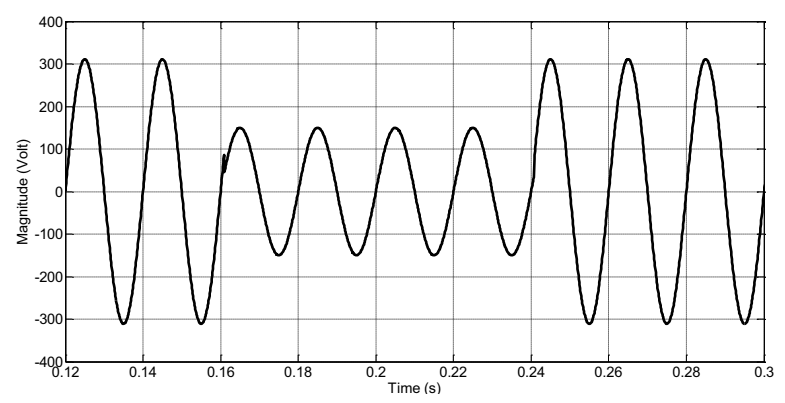

Fig. 5. Single phase PLL input voltage profile

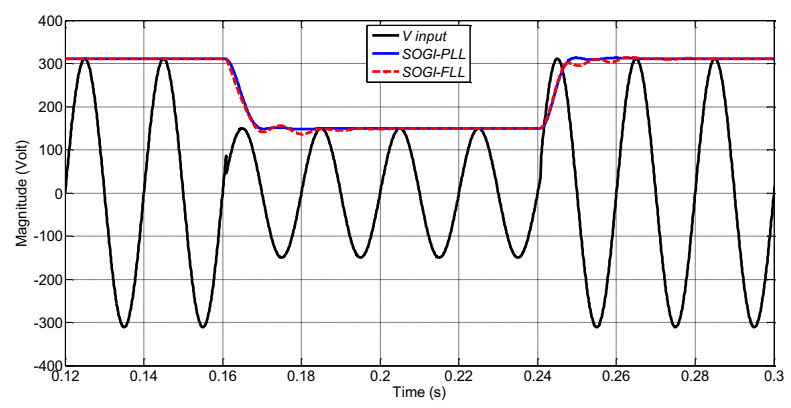

Fig. 6. Results of magnitude detection with SOGI-PLL and SOGI-FLL

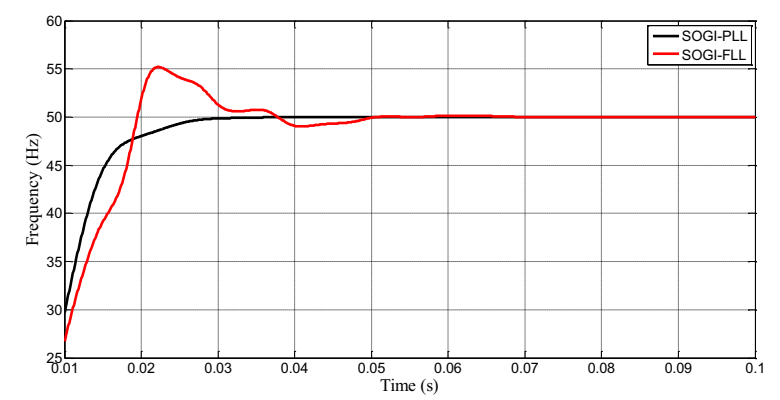

Fig. 7. Results of frequency detection with SOGI-PLL and SOGI-FLL

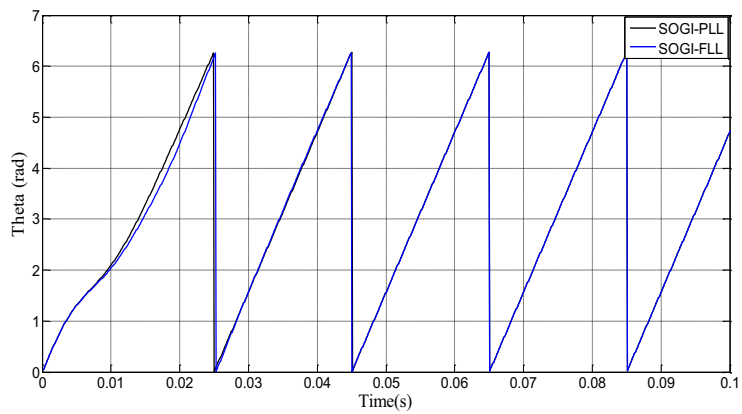

Fig. 8. Results of phase detection with SOGI-PLL and SOGIFLL

As can be seen from the simulation results, the output magnitude, frequency, and phase angle results of detection of SOGI-PLL and SOGI-FLL are entirely accurate even though the grid is changing magnitude, transient waves occur at the magnitude and frequency detection but only at 0 to 0.05 seconds. The magnitude detection shows that the grid is 311 volts $(220 \sqrt{2})$, seconds 0.16 to 0.24 seconds the magnitude decreases to 150 volts, seconds to 0.25 , the magnitude returns to normal. Frequency detection also shows an accurate value of $50 \mathrm{~Hz}$. In addition to the magnitude and frequency, the saw wave-shaped phase with a value of 0 $-6,28 \mathrm{rad}$ represents a value of $0-2 \pi$ which is the size of theta $(\theta)$ also shows an accurate value.

\subsection{The performance of PLL against grid frequency changes}

Fig. 9 shows changes in the frequency of grid detected by SOGI-PLL and SOGI-FLL. Based on fig.9 it appears that the frequency in seconds 0 to 0.15 is $50 \mathrm{~Hz}$, the 0.15 seconds changes in frequency. SOGI-FLL can detect $49 \mathrm{~Hz}$ frequencies and adapt at 0.2 seconds, while SOGIPLL cannot detect frequencies accurately, from Fig. 9 showing frequencies ranging from $48.5 \mathrm{~Hz}$ to $49.5 \mathrm{~Hz}$. Changes in frequency in the grid also affect the estimated magnitude and phase angle, as shown in Fig. 10 and Fig. 11.

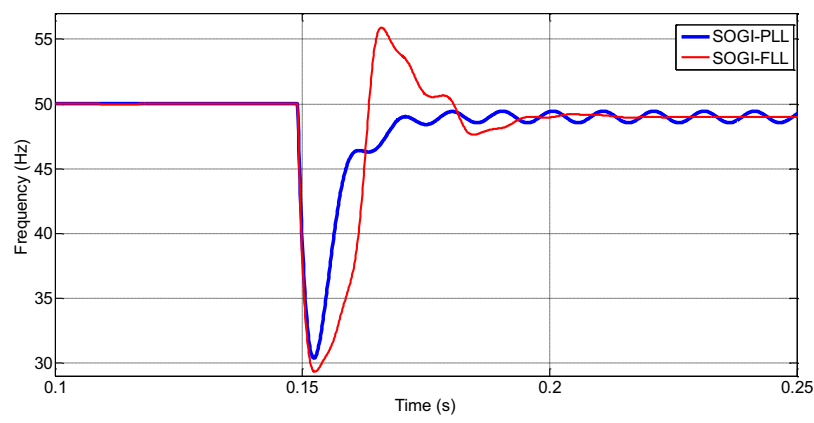

Fig. 9. Results of frequency change detection by SOGI-PLL and SOGI-FLL

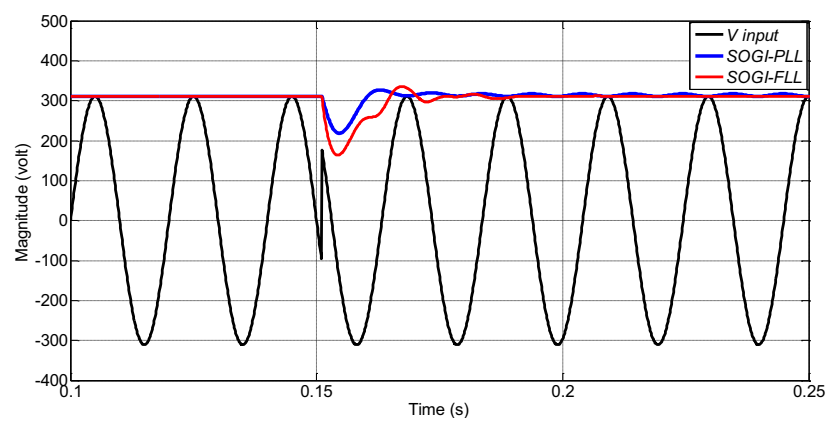

Fig. 10. Results of magnitude detection with SOGI-PLL and SOGI-FLL for electrical voltage profiles that experience changes in frequency

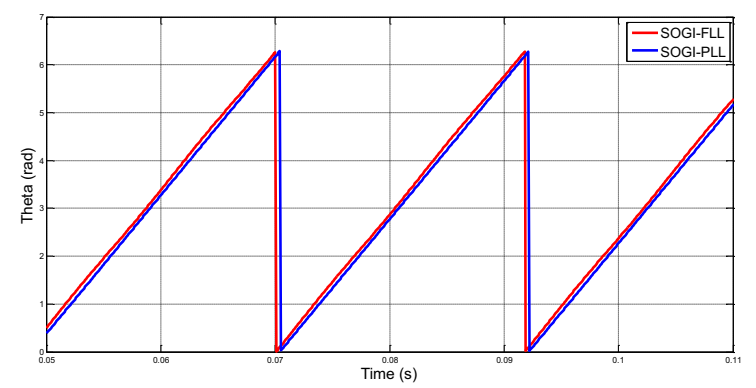

Fig. 11. Phase detection results with SOGI-PLL and SOGIFLL for electrical voltage profiles that experience changes in frequency 
As can be seen from the simulation results, SOGIFLL in its steady state is relatively accurate. Even though under voltage and frequency conditions that change, different results shown at SOGI-PLL output, changes in frequency in the grid cause magnitude detection inaccurate frequency and phase, changing frequency between $48.5 \mathrm{~Hz}$ to $49.5 \mathrm{~Hz}$, magnitudes ranging from 311 volts to 325 volts, as well as phase detection, frequency changes in the grid cause delays in phase detection.

\subsection{The influence of FLL feedback amplifiers on synchronization performance}

Judging from its transient performance, the output of SOGI-FLL in response to changes in frequency and magnitude shows a relatively slow response even though steady-state shows an accurate value, it is necessary to look for FLL feedback gain that has the lowest transient, so that changes in frequency or magnitude as fast can be detected to support synchronization performance.

Gamma $(\gamma)$ as in the SOGI-FLL structure shown in Fig. 4, simulated in Fig. 12 to Fig. 13.

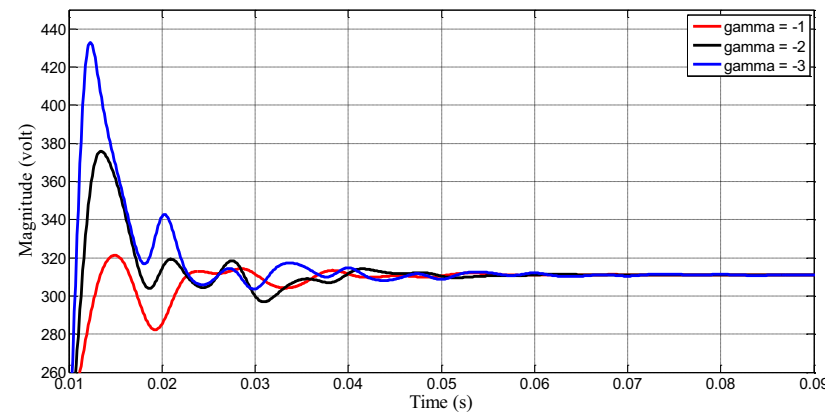

Fig. 12. Comparison of FLL feedback gain against the detection of voltage magnitude

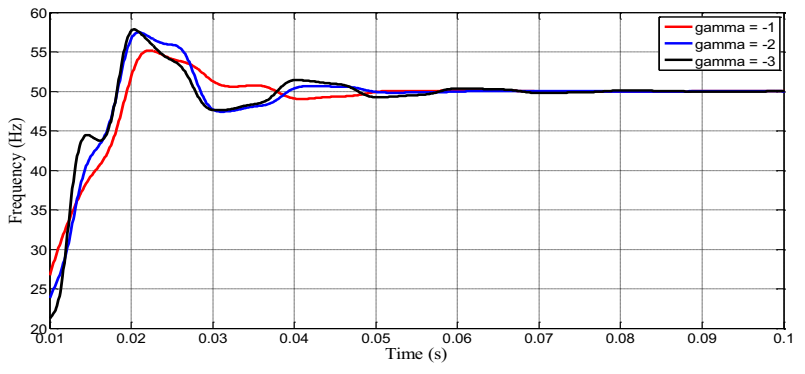

Fig. 13. Comparison of FLL feedback gain against frequency detection

From the simulation shown Fig. 12 the magnitude detected by SOGI-FLL with gamma -1 has an overshoot value of $2.8 \%$ from a magnitude of 311 volts, this value is lowest compared to other FLL feedback gain. Likewise in the frequency detection shown in Fig. 13. The frequency detected by SOGI-FLL has an overshoot value of $10 \%$ of the grid frequency $50 \mathrm{~Hz}$; this value is the lowest compared to other FLL feedback gain. The lower the overshoot value, the better the synchronization system. Fig.14. Shows an FLL feedback gain that has a value greater than -1 . The picture shows inaccurate magnitude detection, as in the SOGI-PLL system.

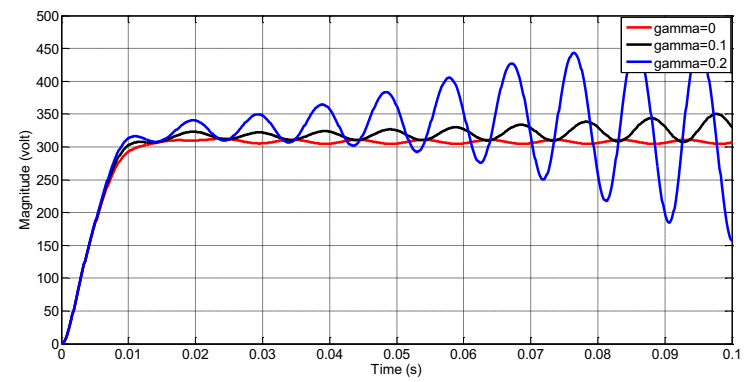

Fig. 14. Effect of FLL feedback gain greater than -1 against magnitude detection

From the simulation shown in Fig. 12. to Fig. 14. it can be concluded that the FLL feedback amplifier that has the best performance is gamma -1, from the simulation, shows the transient wave overshoot value of at least $2.8 \%$, and the system can adapt to changes in the frequency of the grid within 0.05 seconds.

\section{Conclusion}

Based on the simulation studies that have conducted, it appears that SOGI-FLL is superior to SOGI-PLL in a steady state, but in a transient state, SOGI-PLL is superior to SOGI-FLL.

The next topic of this research is the realization of SOGI-FLL into a real-time algorithm on the digital signal controller (DSC) system.

\section{Acknowledgment}

This research was financially supported by the faculty of engineering, Diponegoro University Indonesia trough Strategic Research Grant 2019 with contract number [3161/3/UN7.3.3/PG/2019]

\section{References}

1. Zakzouk, E. Nahla, et al., PV Single-Phase Gridconnected Converter: Dc-Link Voltage Sensorless Prospective, IEEE Journal of Emerging and Selected Topics in Power Electronics 5(1), 526-546 (2017)

2. B.K. Naick, Fuzzy Logic Controller based PV System Connected in Standalone and Grid Connected Mode of Operation with Variation of Load, International Journal of Renewable Energy Research (IJRER) 7(1), 311-322 (2017)

3. A.M. Mustafa, et al, Reliability Assessment of Grid Connected Photovoltaic Generation Systems, Renewable Energy Research and Applications (ICRERA), 2015 International Conference on. IEEE (2015)

4. I. Setiawan, M. Facta, A. Priyadi, M.H. Purnomo, Estimator Parameter Tegangan Jaringan Tiga Fasa Berbasis D-SOGI PLL, Teknologi Elektro, 16(02) (2017) 
5. A.S.N. Mokhtar, M.B.I. Raez, M. Marufuzzaman, M.A.M. Ali, Hardware Implementation of A High Speed Inverse Park Transformation Using Cordic And Pll for Foc Brushless Servo Drive, Elektron. ir Elektrotechnika 19(3), 23-26 (2013)

6. B.M. Lopes, S.M. Silva, R.P. Campana, W.C. Boaventura, B.J.C. Filho, Performance Evaluation of PLL Algorithms for Single-Phase GridConnected Systems, IEEE Industry Applications Society (2004)

7. D.G. Patiño, E.G.G. Erira, E.E. Rosero, J.R. Fuelagán, SOGI-FLL for Synchronization and Fault Detection in An Inverter Connected to the Grid, IEEE PES Innov. Smart Grid Technol. Lat. Am. ISGT LATAM 2015, 833-838 (2016)

8. J.S. Park, T.H. Nguyen, D.C. Lee, Advanced SOGI-FLL Scheme Based on Fuzzy Logic for Single-Phase Grid-Connected Converters. Journal of Power Electronics 14(3), 598-607 (2014)

9. G. Fedele, A. Ferrise, Identification of a Biased Sinusoidal Signal, IEEE transactions on automatic control 57(7) (2012)

10. I. Kopriva, D. Juricic, Performance Evaluation of the Hilbert Transform Based Digital Phase-Locked Loop, Institute for Defense Studies R\&D Bijenicka 46, 10000 Zagreb, Croatia.

11. M. Kumm, M.S. Sanjari, Digital Hilbert Transformers For Fpga-Based Phase-Locked Loops, gesellschaft fur schwerionenforschung (gsi) 64291 darmstadt.

12. I. Kopriva, D. Juricic, Performance Evaluation of the Hilbert Transform Based Digital Phase-Locked Loop, Institute for Defense Studies R\&D, Bijenicka 46, 10000 Zagreb, Croatia.

13. I. Setiawan, T. Andromeda, M. Facta, S. Handoko, Implementation and Performance Analysis of a Single Phase Synchronization Technique based on $T / 4$ Delay PLL, International Journal of Renewable Energy Research 8(1) (2018)

14. M. Ciobotaru, R. Teodorescu, F. Blaabjerg, A New Single-Phase PLL Structure Based on Second Order Generalized Integrator, Proc. IEEE Power Electron. Spec. Conf. (PESC'06), 1-7 (2006)

15. M. H. Abdurrahman, I. Setiawan, and S. Handoko, Design and Implementation of Synchronous Reference Frame-Phase Locked Loop (Srf-Pll) for One Phase Voltage Using DSPIC30F4011, Transient 7(1), Maret (2018)

16. K. Możdżyński, K. Rafał, M. Bobrowska-Rafał, Application of the Second Order Generalized Integrator in Digital Control Systems, Archives of Electrical Engineering 63(3), 423-437 (2014) 